\title{
Main Immunogenic Region Structure Promotes Binding of Conformation-Dependent Myasthenia Gravis Autoantibodies, Nicotinic Acetylcholine Receptor Conformation Maturation, and Agonist Sensitivity
}

\author{
Jie Luo, ${ }^{1}$ Palmer Taylor, ${ }^{2}$ Mario Losen, ${ }^{3}$ Marc H. de Baets, ${ }^{3}$ G. Diane Shelton, ${ }^{4}$ and Jon Lindstrom ${ }^{1}$ \\ ${ }^{1}$ Department of Neuroscience, University of Pennsylvania Medical School, Philadelphia, Pennsylvania 19104-6074, ${ }^{2}$ Department of Pharmacology, Skaggs \\ School of Pharmacy and Pharmaceutical Sciences, University of California, San Diego, La Jolla, California 92093-0650, ${ }^{3}$ Neuroimmunology Group, \\ Department of Neuroscience, School of Mental Health and Neuroscience, Maastricht University, 6200 MD Maastricht, The Netherlands, and ${ }^{4}$ Department of \\ Pathology, School of Medicine, University of California, San Diego, La Jolla, California 92093-0709
}

The main immunogenic region (MIR) is a conformation-dependent region at the extracellular apex of $\alpha 1$ subunits of muscle nicotinic acetylcholine receptor (AChR) that is the target of half or more of the autoantibodies to muscle AChRs in human myasthenia gravis and rat experimental autoimmune myasthenia gravis. By making chimeras of human $\alpha 1$ subunits with $\alpha 7$ subunits, both MIR epitopes recognized by rat mAbs and by the patient-derived human mAb 637 to the MIR were determined to consist of two discontiguous sequences, which are adjacent only in the native conformation. The MIR, including loop $\alpha 167-76$ in combination with the N-terminal $\alpha$ helix $\alpha 11-14$, conferred high-affinity binding for most rat mAbs to the MIR. However, an additional sequence corresponding to $\alpha 1$ 15-32 was required for high-affinity binding of human mAb 637. A water soluble chimera of Aplysia acetylcholine binding protein with the same $\alpha 1$ MIR sequences substituted was recognized by a majority of human, feline, and canine myasthenia gravis sera. The presence of the $\alpha 1$ MIR sequences in $\alpha 1 / \alpha 7$ chimeras greatly promoted AChR expression and significantly altered the sensitivity to activation. This reveals a structural and functional, as well as antigenic, significance of the MIR.

\section{Introduction}

Myasthenia gravis (MG) and experimental autoimmune myasthenia gravis (EAMG), are caused by antibody-mediated autoimmune responses to nicotinic acetylcholine receptors (AChRs) which impair neuromuscular transmission (Lindstrom, 2000). At least half of the autoantibodies in both MG and EAMG are directed at the main immunogenic region (MIR) on AChR $\alpha 1$ subunits (Tzartos et al., 1998). The MIR is defined by the ability of a single rat mAb to inhibit binding of many autoantibodies from MG patients or rats with EAMG (Tzartos and Lindstrom, 1980; Tzartos et al., 1982, 1983). The $\alpha 1$ sequence $66-76$, the MIR loop, is crucial to the MIR (Gullick and Lindstrom, 1983; Das and Lindstrom, 1989; Saedi et al., 1990; Tzartos et al., 1990; Tzartos et al., 1998). The antigenicity and myasthenogenicity of the MIR depend greatly on the native conformation of the AChR

Received June 15, 2009; revised Sept. 3, 2009; accepted Sept. 7, 2009.

This research was supported by National Institutes of Health Grants NS11323 and NS052463 to J.L. and U01 DA019372 and GM 18360 to P.T. This work was also supported by grants from the Prinses Beatrix Fonds, L'Association Française contre les Myopathies and the MYASTAID project grant of the Sixth Framework Programme of the European Community to M.d.B. We thank Barbara Campling for comments on this manuscript.

Correspondence should be addressed to Dr. Jon M. Lindstrom, Department of Neuroscience, University of Pennsylvania Medical School, 217 Stemmler Hall, 36th and Hamilton Walk, Philadelphia, PA 19104-6074. E-mail: jslkk@mail.med.upenn.edu.

DOI:10.1523/JNEUROSCI.2833-09.2009

Copyright $\odot 2009$ Society for Neuroscience $\quad$ 0270-6474/09/2913898-11\$15.00/0
(Lindstrom et al., 1978; Lindstrom and Einarson, 1979; Lennon et al., 1991; Im et al., 2000).

mAbs to the MIR can passively transfer EAMG into experimental animals (Tzartos et al., 1987; van der Neut Kolfschoten et al., 2007). These mAbs exhibit the primary pathological activities of serum antibodies: complement-dependent focal lysis of the postsynaptic membrane (which destroys AChRs and disrupts synaptic morphology) and antigenic modulation (which reduces the number of AChRs through crosslinking AChRs and thereby increasing their internalization). The orientation of the MIR at the outer perimeter and away from the central axis of the AChR explains why mAbs to the MIR are very effective at crosslinking adjacent AChRs and triggering antigenic modulation (Conti-Tronconi et al., 1981; Beroukhim and Unwin, 1995).

Antibody competition experiments reveal that the antibody repertoire in MG patients is similar to that in EAMG rats immunized with purified AChRs (Tzartos et al., 1998). Like human MG, canine MG also has a high proportion of autoantibodies to the MIR (Shelton et al., 1988). Some rat mAbs to the MIR also bind in a conformation-dependent manner to human neuronal $\alpha 3, \alpha 5$, and $\beta 3$ subunits (Kuryatov et al., 1997; Wang et al., 1998), but autoantibodies from MG patient sera do not react with human neuronal AChRs (Vernino and Lennon, 2004). This implies that these rat $\mathrm{mAbs}$ and human $\mathrm{MG}$ autoantibodies recognize different epitopes within the MIR. 
Table 1. Properties of mAbs to the MIR used in this study

\begin{tabular}{|c|c|c|c|c|c|c|}
\hline \multirow[b]{2}{*}{$\mathrm{mAb}$} & \multirow[b]{2}{*}{ Immunogen } & \multirow[b]{2}{*}{ Species immunized } & \multicolumn{3}{|c|}{ Specificity } & \multirow[b]{2}{*}{ Comments } \\
\hline & & & Subunit & Epitope & Original description & \\
\hline 35 & Electrophorus AChR & Rat & $\alpha 1$ & MIR & Tzartos et al., 1981 & $\begin{array}{l}\text { Does not bind well to } \alpha 1 \text { on Western blots (Das and Lindstrom, 1989). Binds native } \alpha 1 \\
\quad \text { from all species tested but Xenopus. Passively transfers EAMG (Tzartos et al., 1987). }\end{array}$ \\
\hline 192 & $\begin{array}{l}\text { Purified human muscle } \\
\quad \text { AChR }\end{array}$ & Rat & $\alpha 1$ & MIR & Tzartos et al., 1983 & $\begin{array}{l}\text { Binds with an affinity of } 1 \times 10^{-11} \mathrm{M} \text { to native human } \alpha 1 \mathrm{AChR} \text {, but not to denatured } \\
\text { human } \alpha 1 \text { (Tzartos et al., 1998). Its affinity for rat AChR is very low }\left(6.5 \times 10^{-7} \mathrm{M}\right) \text {. }\end{array}$ \\
\hline 198 & $\begin{array}{l}\text { Purified human muscle } \\
\text { AChR }\end{array}$ & Rat & $\alpha 1$ & MIR & Tzartos et al., 1983 & Binds both native and denatured $\alpha 1$ (Das and Lindstrom, 1989). Passively transfers EAMG. \\
\hline 210 & $\begin{array}{l}\text { Bovine and mouse } \\
\text { AChR }\end{array}$ & Rat & $\alpha 1$ & MIR & Tzartos et al., 1987 & $\begin{array}{l}\text { Binds native human } \alpha 1, \alpha 3, \alpha 5, \beta 3 \text { (Kuryatov et al., 2008). Binds } \alpha 1 \text { on Western blots. } \\
\text { Passively transfers EAMG (Tzartos et al., 1987). }\end{array}$ \\
\hline 637 & & $\begin{array}{l}\text { Derived from thymus } \\
\text { of a MG patient }\end{array}$ & $\alpha 1$ & MIR & Graus et al., 1997 & $\begin{array}{l}\text { Binds native human } \alpha 1 \text { competitively with rat mAbs to the MIR (Graus et al., 1997). } \\
\text { Passively transfers MG to monkeys (van der Neut Kolfschoten et al., 2007). }\end{array}$ \\
\hline
\end{tabular}

Here, we precisely mapped MIR epitopes recognized in MG or EAMG by making chimeras in which sequences of human muscle $\alpha 1$ subunits replaced parts of the human neuronal $\alpha 7 \mathrm{AChR}$ or Aplysia ACh binding protein (AChBP). Two sequences, which were adjacent only in the native $\alpha 1$ conformation, formed the MIR, thereby explaining conformation dependence of the MIR. The great influence of the $\alpha 1$ MIR sequences on AChR expression and sensitivity to activation implies important roles of the MIR in conformation changes associated with subunit conformational maturation and assembly as well as AChR activation.

\section{Materials and Methods}

$\alpha 7$ chimeras. Human $\alpha 7$ cDNA was subcloned into the BglII site of the PMXT vector as previously described (Peng et al., 1994). The sequences of $\alpha 72-14$ and 66-76 were substituted with homologous human $\alpha 1$ sequences by multiple-step PCR using appropriate pairs of forward and reverse synthetic oligonucleotide primers (Invitrogen). The extra $\alpha 1$ sequences $60-65$ and 77-81 substituted for corresponding sequences of the above chimera using a similar approach to express an extended MIR loop.

To incorporate the $\alpha 1$ sequence 1-32, we engineered a BamH I site and a BstE II site at each end of the target sequence in $\alpha 7 \mathrm{cDNA}$, or the chimera with the extended MIR loop. Using a similar approach, we introduced a BamH I site between the sequences coding for signal peptide and $\mathrm{N}$ terminus of $\alpha 1$ subunit in $\alpha 1 \mathrm{cDNA}$ by PCR. The mutated $\alpha 1$ and $\alpha 7$ cDNA clones were digested with BamH I and BstE II, and a purified 212-bp fragment from $\alpha 1 \mathrm{cDNA}$ was ligated together with the remaining fragments of $\alpha 7$ into the PMXT vector.

Chimera cDNAs were checked for accuracy by DNA sequencing before cRNA preparation. cRNAs were synthesized in vitro using the SP6 mMessage mMachine kit (Ambion). Schematic diagrams of the wildtype subunits and chimeras are shown in Figure $1 B$.

$A C h B P$ chimeras. A KpnI site was engineered at position Phe 35 of a cDNA encoding the Aplysia acetylcholine binding protein $(A-\mathrm{AChBP})$ in the FLAG-CMV-3 expression vector (Hansen et al., 2002). A 123-bp PCR product coding for the N-terminal sequence $\alpha 11-30$ (Val 31 and Thr 32 are conserved between $\alpha 1$ subunit and A-AChBP) was produced using the full-length $\alpha 1$ cDNA clone in TE1.1 as a template. The upstream and downstream sequences were constructed to contain HindIII and KpnI restriction sites respectively. Both the purified PCR product and $A$-AChBP cDNA were cut with the restriction enzymes HindIII and KpnI. A fragment coding for $\alpha 11-30$ was ligated together with the remaining fragments of $A$-AChBP. Then the KpnI site was removed by PCR.

To graft the MIR loop $\alpha 160-81$ into the above chimera, another KpnI site was introduced at position Lys 61 by two-step PCR. An 87-bp PCR product encoding $\alpha 160-81$, which was engineered to have a KpnI site at $5^{\prime}$-end and a EcoR V site at $3^{\prime}$-end, was produced from the full-length $\alpha 1$ cDNA. The purified PCR product was cut with the restriction enzymes $\mathrm{KpnI}$ and EcoR V for ligation with the remaining fragments of chimeric $\alpha 1(1-30) / A C h B P$, which was cut with the same pair of enzymes. Then the second KpnI site was removed by PCR. $m A b s$. Monoclonal anti-FLAG M2 antibody was purchased from Sigma-Aldrich. All subunit-specific mAbs used here have been characterized and described previously (Lindstrom, 1996). Properties of mAbs to the MIR used are summarized in Table 1. mAb 306 and mAb 319 are directed at the cytoplasmic domain of $\alpha 7$ (McLane et al., 1992).

$M G$ patient sera. Serum from human MG patients was provided by archived samples from the Lindstrom lab and the de Baets lab. Sera from canine and feline MG patients were provided from the Shelton lab and, chosen to be $\geq 0.6 \mathrm{~nm}$ titer for canines and $\geq 0.3 \mathrm{~nm}$ titer for felines.

Xenopus oocyte expression. Oocytes were prepared for microinjection as described by Colman (1984), and injected with $50 \mathrm{ng}$ of cRNA of each of wild-type or chimeric $\alpha 7$ subunits unless otherwise specified. They were incubated for 3-4 d after injection in a modified L-15 medium containing 50\% Leibovitz L-15 (Invitrogen), 10 mм HEPES, pH 7.5, 10 unit $/ \mathrm{ml}$ penicillin, and $10 \mathrm{mg} / \mathrm{ml}$ streptomycin at $18^{\circ} \mathrm{C}$. Surface expression was determined by incubating oocytes in L-15 medium containing $1 \%$ BSA and $4 \mathrm{~nm}^{125} \mathrm{I} \alpha \mathrm{Bgt}$ for $1 \mathrm{~h}$ at room temperature followed by washing steps with L-15 medium. Nonspecific binding was determined by incubating noninjected oocytes under the same conditions.

Radioimmunoassay. On day 5 after injection, groups of oocytes were homogenized in buffer A ( $50 \mathrm{~mm} \mathrm{Na} \mathrm{HPO}_{4}-\mathrm{NaH}_{2} \mathrm{PO}_{4}, 50 \mathrm{~mm} \mathrm{NaCl}, 5$ mм EDTA, 5 mм EGTA, 5 mm benzamidine, 15 mm iodoacetamide, $2 \mathrm{~mm}$ phenylmethylsulfonyl fluoride, $\mathrm{pH} 7.5$ ). Cell membranes were pelleted by centrifugation at $15,000 \times g$ for $20 \mathrm{~min}$, followed by solubilizing in buffer A containing 2\% Triton X-100 for $1 \mathrm{~h}$ at room temperature. After cellular debris was removed, aliquots of oocyte extract were incubated with appropriate amounts of a $5 \mathrm{mg} / \mathrm{ml}$ stock of mAbs with $5 \mu$ l of normal serum and $10 \mathrm{nM}{ }^{125} \mathrm{I} \alpha$ Bgt overnight at $4^{\circ} \mathrm{C}$. AChR-antibody complexes were precipitated using $40 \mu \mathrm{l}$ of a standardized stock of goat anti-human IgG (for human IgG) or $100 \mu \mathrm{l}$ of a standardized stock of sheep anti-rat IgG (for rat $\mathrm{mAbs}$ ) for $2 \mathrm{~h}$ at room temperature. This precipitate was pelleted by centrifugation and washed two times with $1 \mathrm{ml}$ of $0.5 \%$ Triton X-100 in PBS (10 mm sodium phosphate buffer, $100 \mathrm{~mm} \mathrm{NaCl}, \mathrm{pH} 7.5$ ). The pellets were assayed in a $\gamma$ counter.

Electrophysiological recordings. Currents in oocytes were measured using a standard two-microelectrode voltage-clamp amplifier setup (oocyte clamp OC-725, Warner Instrument) as previously described (Gerzanich et al., 1998). Dose-response curves were derived by determining the maximum ACh response obtainable on each oocyte, then normalizing these responses as a fraction of the maximum response. Normalized responses from several oocytes were analyzed using the Hill equation $\left(R=1 /\left(1+10^{\left.\left(\operatorname{LogEC}_{50}-\log [\mathrm{ACh}]\right) \times \text { Hill slope }\right)}\right)\right.$ and the curvefitting program Kaleidagraph (Synergy) to determine the $\mathrm{EC}_{50}$ values reported.

Western blots. The groups of oocytes were homogenized in buffer A containing $10 \mu \mathrm{g} / \mathrm{ml}$ DNase followed by three freezing/melting cycles. Cell membranes were pelleted by centrifugation and resuspended in $1 \mathrm{ml}$ of buffer A containing $10 \mu \mathrm{g} / \mathrm{ml}$ DNase, followed by an $1 \mathrm{~h}$ incubation at room temperature. Membrane fractions were collected by centrifugation and solubilized in buffer A containing 2\% Triton X-100 for $1 \mathrm{~h}$ at room temperature. After cellular debris was removed, aliquots of oocyte extract (corresponding to one oocyte) were separated on pre-cast NuPAGE 10\% Bis-Tris Gels (Invitrogen). The transfers were conducted in a SemiPhor 
semi-dry electroblotting chamber (Hoefer) to Trans-Blot Medium PVDF membrane (Bio-Rad). The blots were quenched using $5 \%$ dried milk after transfer for $80 \mathrm{~min}$. The subunits were detected by a 1:1 mixture of mAbs 306 and 319 .

Expression and purification of chimeric AChBP. HEK293S cells lacking the $N$-acetylglucosaminyltransferase I $\left(\mathrm{GnTI}^{-}\right)$gene (Reeves et al., 2002) were maintained in DMEM (high glucose) (Invitrogen) supplemented with $10 \%$ fetal bovine serum (Thermo Fisher Scientific), 100 units $/ \mathrm{ml}$ penicillin, $100 \mu \mathrm{g} / \mathrm{ml}$ streptomycin, and $2 \mathrm{~mm}$ L-glutamine (all from Invitrogen) in a $\mathrm{CO}_{2}(10 \%)$ incubator. Wild-type $\mathrm{AChBP}$ or chimeric $\alpha 1(1-30,60-81) / A C h B P$ with a N-terminal FLAG tag were transfected into HEK293S(GnTI ${ }^{-}$) cells using the FuGene6 transfection agent (Roche Diagnostics), followed by selection with G418 to yield stable cell lines secreting AChBP. Both wild-type AChBP and chimera were expressed as a soluble exported protein. Culture media containing AChBP or chimera were collected and applied onto a FLAG antibody column. Elution with the $3 \times$ FLAG peptide yielded purified proteins (Hansen et al., 2004).

Immobilization of chimeric AChBP on Activated $C H$ Sepharose $4 B$. $\alpha 1(1-30,60-81) / A C h B P$ chimera was immobilized on Activated $\mathrm{CH}$ Sepharose $4 \mathrm{~B}$ at a protein/resin ratio of $2 \mathrm{mg} / \mathrm{ml}$ according to the protocol recommended by the manufacturer (GE Healthcare). The prepared resin was stored in PBS, pH 7.4, containing 0.05\% sodium azide. In parallel, an equal amount of bovine serum albumin (BSA) was immobilized on Activated $\mathrm{CH}$ Sepharose $4 \mathrm{~B}$ under the same conditions as a control.

Immunoadsorption of anti-MIR autoantibodies. Twenty-five microliters of the $\alpha 1(1-30,60-81) / \mathrm{AChBP}$ chimera beads or BSA beads were incubated overnight at $4^{\circ} \mathrm{C}$ with $70 \mu \mathrm{l}$ of diluted MG sera in PBS, $0.2 \%$ BSA containing $35 \mathrm{fmol}$ of anti-AChR antibodies in a compact reaction column (USB), followed by centrifugation and a wash with $70 \mu \mathrm{l}$ of PBS, $0.2 \%$ BSA. Combined supernatants were assayed for unbound anti$\mathrm{AChR}$ antibodies using a radioimmunoassay described as above with a substitution of TE671 AChR (transfected with $\varepsilon$ subunit) for chimeric AChR. The percentage of immunoadsorption was calculated as follows: $\%$ immunoadsorption $=[1-($ anti-AChR titers after adsorbed with chimera beads) $/($ anti-AChR titers after adsorbed with BSA beads $) \times 100$.

$m A b 35$ competition assay. Anti-AChR titers of MG sera were determined using a radioimmunoassay as above in the presence or absence of $50 \mathrm{~nm}$ of mAb 35. The immune complex of ${ }^{125} \mathrm{I}-\alpha$ Bgt labeled AChR and MG autoantibody was precipitated using goat anti-human IgG depleted with normal rat serum. The percentage of inhibition was calculated as follows: $\%$ inhibition $=[1-($ anti-AChR titers in the presence of $\mathrm{mAb}$ $35) /($ anti-AChR titers in the absence of $\mathrm{mAb} 35) \times 100$.

Statistics. Student's two tailed $t$ test was used to determine the significance of differences between group means. All data represent the mean $\pm \mathrm{SD}$.

\section{Results}

\section{Mapping the antigenic structure of the MIR}

Sequences of the extracellular domain of human AChR $\alpha 7$ subunits were replaced by homologous human $\alpha 1$ sequences to determine which $\alpha 1$ sequences would confer antigenicity of an $\alpha 1$ MIR to an $\alpha 1 / \alpha 7$ chimera (Fig. 1). Properties of the rat and human mAbs to the MIR used in his study are summarized in Table 1. Rat mAb 210 to the MIR binds both native and denatured $\alpha 1$ (Lindstrom, 2000). Incorporating only the putative MIR loop $\alpha 166-76$ into $\alpha 7$ allowed binding of mAb 210 (Table 2 ), but at only $1 / 500$ th of the affinity with which it bound native AChR. mAb 198 had similarly low affinity for this chimera compared to native AChR. The $\alpha 1(66-76) / \alpha 7$ chimera was not bound by the absolutely conformation-dependent mAb 35 . Increasing the length of the $\alpha 1$ insert to 60-81 increased the binding affinities of mAb 210 and mAb 198 by 227- and 79-fold, respectively. The $\alpha 1(60-81) / \alpha 7$ chimera was bound by mAb 35, but with only $1 / 1000$ th the affinity for native AChR (Table 2).

Examination of the structure of Aplysia AChBP revealed that the N-terminal $\alpha$ helical sequence 1-14 paralleled the putative MIR loop 66-76 (Fig. 1A). $\alpha 1 / \alpha 7$ chimeras containing both the $\alpha 1 \mathrm{~N}$-terminal $\alpha$ helix and the MIR loop were efficiently bound by mAbs with sub-nanomolar affinities (Table 2). The contribution to the MIR of the two segments of the extracellular domain of the $\alpha 1$ subunit, which are adjacent only in the native conformation, explains the conformation dependence of the binding of these mAbs to the MIR. The presence of $\alpha 12-14$ along with $\alpha 1$ $60-81$ increased the affinity with which mAb 210 was bound 2600 -fold. Then the binding affinity of mAb 210 for the chimera exceeded that for muscle AChR by fourfold. This may be because the chimera is a homopentamer, which may permit both $\mathrm{mAb}$ binding sites to bind within a single pentamer, whereas in muscle type AChRs the two $\alpha 1$ MIRs are oriented so that they cannot be crosslinked by a single mAb (Conti-Tronconi et al., 1981; Beroukhim and Unwin, 1995). The affinity of mAb 198 for this chimera was also greater than for native AChR. The presence of $\alpha 12-14$ had the greatest effect on the conformation-dependent mAb35, increasing the affinity with which it was bound 91,000fold, also to greater than for native AChR. However, the above chimeras bound to neither rat mAb 192 nor human mAb 637. This suggests that some human autoantibodies to the MIR bind to epitopes distinct from those which are recognized by most mAbs to the MIR from rats with EAMG, but close enough to compete for binding to human muscle AChRs (Tzartos and Lindstrom, 1980). The ring in Figure $1 A$ showing the area on a protein typically obscured by a bound antibody illustrates how several adjacent epitopes could be obscured by a single bound mAb. Comparison of the size of an Fab fragment of an IgG molecule to the size of the $\alpha 1$ extracellular domain further emphasizes this point (Fig. $1 B$ ).

mAb 192 depends absolutely for its binding on the native conformation of $\alpha 1$, and binds to rat AChRs with 10,000-fold lower affinity than to human AChRs (Tzartos et al., 1998). It seems likely that its epitope includes sequences within $\alpha 123-30$, because these are the only nonconservative sequence differences between human and rat anywhere near the MIR (Fig. 1C). An $\alpha 7$ chimera containing both the $\alpha 11-32$ and the longer $60-81$ version of the MIR loop was bound by the human mAb 637 with sub-nanomolar affinity, although it was not recognized by mAb 192 (Table 2). These results emphasize the importance of both AChR sequence and conformation to binding affinity of antibodies.

\section{$\alpha 1$ MIR increases expression of $\alpha 1 / \alpha 7$ chimeras}

Rather than reducing expression of $\alpha 1 / \alpha 7$ chimeras, as one might expect from disruption due to a combination of muscle and neuronal AChR sequences, the chimeras exhibited $>10$ fold, on the average, increased expression on the oocyte surface compared to wild-type $\alpha 7$ (Fig. $2 A$ ). The $\alpha 1(2-14,60-81) / \alpha 7$ chimera showed a 53-fold increase over wild-type $\alpha 7$ on the oocyte surface. This suggests that interactions between the MIR loop and the $\mathrm{N}$-terminal $\alpha$ helix, which produce epitopes recognized with highest affinity by mAbs to the MIR, may also provoke conformational maturation of the $\alpha 1 / \alpha 7$ subunits. In contrast, incorporating only the $\mathrm{N}$-terminal sequence $\alpha 12-14$ or $1-32$ into $\alpha 7$ completely prevented expression of mature AChR on the oocyte surface (Fig. $2 A$ ) or within the oocyte (data not shown). Thus, interactions between the $\mathrm{N}$-terminal sequence and the MIR loop must occur when both are from $\alpha 1$, which make the $\alpha 1$ $\mathrm{N}$ terminal sequences tolerable within the chimera. However, incompatible interactions between $\mathrm{N}$-terminal $\alpha 1$ sequences and the $\alpha 7$ MIR loop may prevent conformational maturation, thereby preventing assembly and the formation of binding sites for ${ }^{125} \mathrm{I} \alpha$ Bgt. Sequences within $\alpha 115-32$ must be poorly compatible with parts of $\alpha 7$ and lead to reduced expression (Fig. 2A). 
A

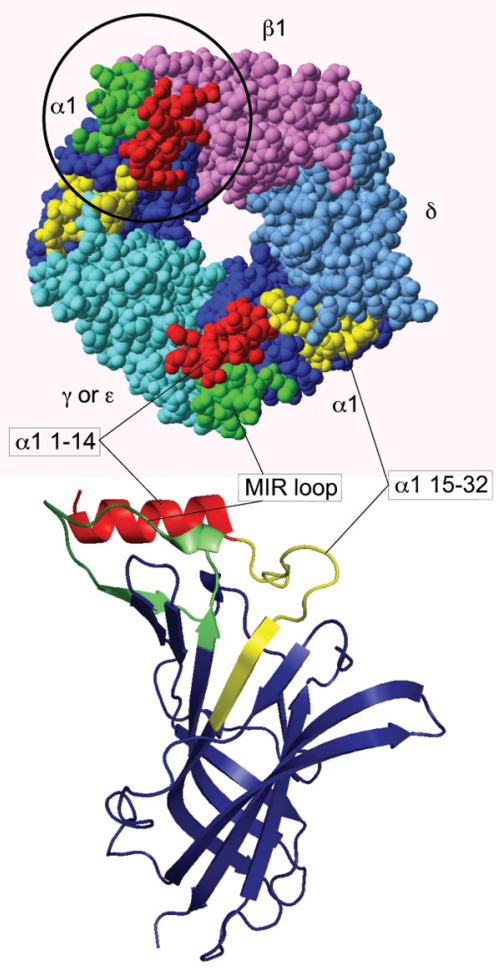

B

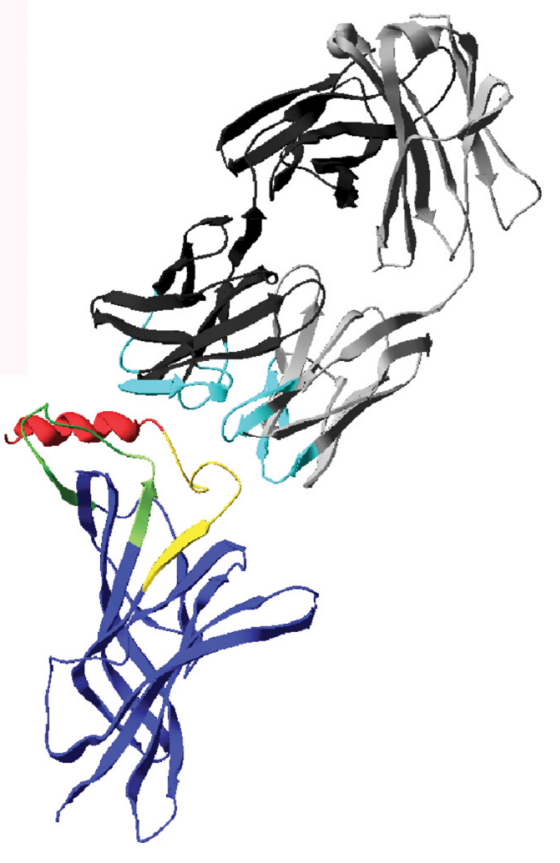

C

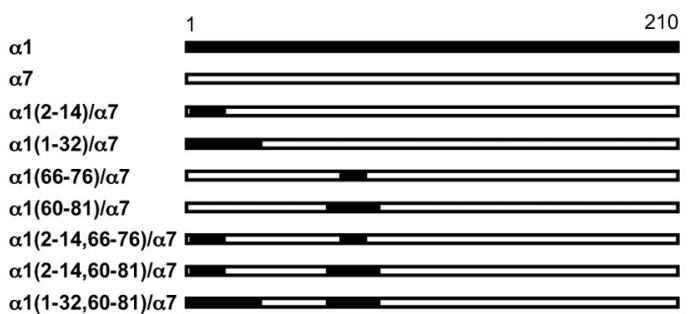

D

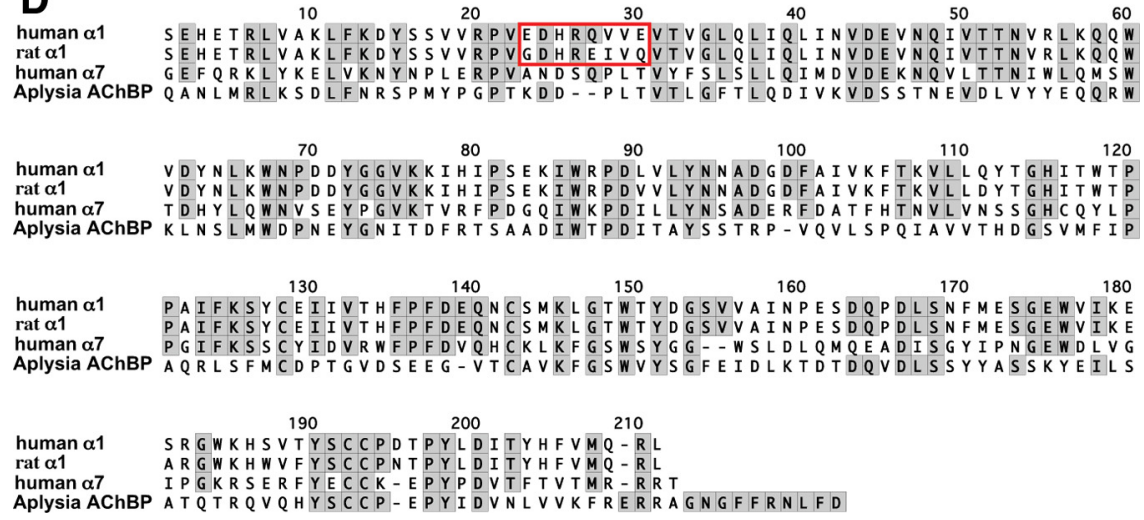

Figure 1. Structures of MIR chimeras. A, Putative MIR components are highlighted on the crystal structure of an Aplysia AChBP subunit (Hansen et al., 2005). A top-view space-filling model is shown at the top. A front-view ribbon diagram, rotated to permit viewing of the MIR from its underside as a prominent appendage, is shown at the bottom. Red highlights the $\alpha$ helical ribbon corresponding to $\alpha 11-14$. The thin segment proceeding the $\alpha$ helix represents a FLAG tag on the AChBP constructs. Yellow highlights sequence corresponding to $\alpha 115-32$. Green highlights sequence corresponding to $\alpha 160-81$, including the MIR loop $\alpha 166-76$. Dark blue designates the remainder of the $\alpha 1$ subunit. A circle with a radius of $15.5 \AA$ and center at the MIR indicates the area typically buried upon antibody binding to a protein antigen (Mariuzza et al., 1987; Konstantakaki et al., 2007). The prominent loop on the right is the ( loop which closes over the ACh binding site when agonists are bound. $B$, The crystal structure of a Fab of mAb 192 (Kontou et al., 2000) is accompanied by the mouse $\alpha 1$ extracellular domain (Dellisanti et al., 2007). This allows comparison between the similar structures of the $\alpha 1$ extracellular domain and AChBP subunit and it allows comparison between the size of an Fab and the MIR. This makes it evident that while very small differences in the sequence and conformation of epitopes can profoundly influence the affinity with which antibodies are bound, the large size of bivalent lgG molecules can result in competitive binding between different closely spaced epitopes within the MIR. Parts of the MIR are highlighted in the same colors as in the
Similar amounts of wild-type and chimeric subunits were detected on a Western blot when equal amounts of cRNAs were injected in Xenopus oocytes (Fig. 2 B, top). Both mAbs 306 and 319 used for the blot are directed at the large cytoplasmic domain of $\alpha 7$ subunit (McLane et al., 1992), thus their binding to $\alpha 7$ should not be affected by mutations of the extracellular domain. It is striking that the same large amount of denatured $\alpha 7$ protein is found in oocytes expressing wild-type $\alpha 7$, $\alpha 1(1-32) / \alpha 7$ chimeras which assemble no ${ }^{125} \mathrm{I} \alpha$ Bgt binding sites of mature AChRs, and $\alpha 1(1-32,60-81) / \alpha 7$ chimeras which assemble 8.3-fold more mature AChRs than wild type. This shows that the great increase in mature AChRs resulting from expression of $\alpha 1 / \alpha 7$ chimeras is caused by increased assembly rather than by increased synthesis of subunits. Even with wild-type $\alpha 7$, assembly is very inefficient (6.6\%). Adsorption with $\alpha$ Bgt beads reveals that $71 \%$ of $\alpha 1(2-14,60-81) / \alpha 7$ chimeras were assembled into pentameric AChRs (Fig. 2C). Conformational maturation of subunits before assembly is probably what limits formation of mature $\alpha 7$ AChRs, as is the case for muscle AChRs (Merlie and Lindstrom, 1983). The MIR, and its interactions with the N-terminal sequences which produces high affinity MIR epitopes, may also be responsible for nucleating conformational maturation of $\alpha 7$ subunits that permits greatly increased assembly of mature AChRs.

\section{Functional chimeric $\alpha 7$ AChRs expressed in oocytes}

$\alpha 1 / \alpha 7$ chimeras retain their ability to function as AChRs, as shown in Figure 3. Presence of the MIR sequences greatly influences the sensitivity to activation of chimeric AChRs by acetylcholine (ACh). The $\alpha 1(1-32,60-81) / \alpha 7$ chimera was tenfold

\footnotetext{
AChBP subunit. The light chain of the Fab is gray and the heavy chain is black. Six hypervariable loops, which form the antigen binding sites, are highlighted in cyan. This mAb to the MIR does not bind to the MIR loop but competes for binding with mAbs which do. The Fab is angled to suggest this, but not actually docked on the subunit. Future studies in which the crystal structures of MIR chimeras with Fabs bound are determined should reveal the contact amino acids involved in binding of mAbs to the MIR. C, A schematic diagram of the $\alpha 1 / \alpha 7$ chimeras used to map MIR epitopes is shown. The extracellular domain of each chimeric subunit is displayed with the $\alpha 1$ sequences indicated by black boxes. $D$, Alignment of extracellular domain sequences of human and rat muscle AChR $\alpha 1$ subunits. Rodent $\alpha 1$ differs significantly at the sequence $\alpha 1$ 23-30 from human $\alpha 1$ (in red), making this sequence a likely candidate for contributing to MIR epitopes which differ between rats and humans, such as the epitope for mAb 192.
} 
Table 2. Dissociation constants $\left(K_{\mathrm{D}}\right)$ of $\mathrm{mAbs}$ to the MIR for $\alpha 1 / \alpha 7$ and $\alpha 1 / \mathrm{AChBP}$ chimeras

\begin{tabular}{|c|c|c|c|c|c|c|c|c|}
\hline \multirow[b]{2}{*}{ mAbs } & \multicolumn{8}{|l|}{$K_{\mathrm{D}}(\mathrm{nm})$} \\
\hline & $\alpha 1 \mathrm{AChR}$ & $\alpha 1(66-76) / \alpha 7$ & $\alpha 1(60-81) / \alpha 7$ & $\begin{array}{l}\alpha 1(2-14,66-76) / \\
\alpha 7\end{array}$ & $\begin{array}{l}\alpha 1(2-14,60-81) / \\
\alpha 7\end{array}$ & $\begin{array}{l}\alpha 1(1-32,60-81) / \\
\alpha 7\end{array}$ & $\begin{array}{l}\alpha 1(1-30,60-81) / \\
\text { AChBP }\end{array}$ & $\begin{array}{l}\alpha 1(1-30,60-81,107-115) / \\
\text { AChBP }\end{array}$ \\
\hline Rat 210 & $0.127 \pm 0.028$ & $80.2 \pm 54.3$ & $0.354 \pm 0.111$ & $0.028 \pm 0.012$ & $0.031 \pm 0.005$ & $0.050 \pm 0.007$ & $0.169 \pm 0.020$ & $0.18 \pm 0.02$ \\
\hline Rat 198 & $12.7 \pm 1.5$ & $3860 \pm 1570$ & $48.9 \pm 40.1$ & $0.109 \pm 0.037$ & $0.068 \pm 0.013$ & $0.183 \pm 0.097$ & $2.50 \pm 0.15$ & $3.19 \pm 0.14$ \\
\hline Rat 35 & $2.06 \pm 1.15$ & None & $2370 \pm 820$ & $0.306 \pm 0.717$ & $0.026 \pm 0.016$ & $0.034 \pm 0.010$ & $0.140 \pm 0.036$ & $0.297 \pm 0.010$ \\
\hline Rat 192 & $0.0071 \pm 0.0007$ & None & None & None & None & None & $0.137 \pm 0.025$ & $0.121 \pm 0.014$ \\
\hline Human 637 & $0.0050 \pm 0.0012$ & None & None & None & None & $0.030 \pm 0.023$ & $0.075 \pm 0.013$ & $0.090 \pm 0.016$ \\
\hline
\end{tabular}

Binding of different concentrations of mAbs to ${ }^{125} \mathrm{I} \alpha$ Bgt labeled $\alpha 1 / \alpha 7$ chimeras or ${ }^{125}$ I labeled $\alpha 1 /$ AChBP chimeras was determined by radioimmunoassay. The averages of duplicate determinations were fitted by the Hil equation $\left(R=1 /\left(1+10^{\text {(LogK } D-\log [A C h]) \times \text { Hill slope) })}\right.\right.$ to determine $K_{D}$ values. The single-fit errors are shown

more sensitive than wild-type $\alpha 7$ to activation by ACh. On the other hand, the $\alpha 1(2-14,60-81) / \alpha 7$ chimera was 13 -fold less sensitive than wild-type $\alpha 7$. These marked changes in sensitivity to activation by ACh were not accompanied by significant change in agonistinduced current per AChR (Fig. 3B). This suggests that the differences between chimeras resulted from differences in probability of channel opening, rather than duration of channel opening due to altered rates of desensitization or altered channel conductance.

Response kinetics are similarly rapid for $\alpha 7$ and the much more sensitive $\alpha 1(1-32,60-81) / \alpha 7$ chimera (Fig. 3C). This shows that the greater sensitivity does not result from greatly decreased desensitization but instead, most likely, from a greater probability of being opened when liganded. The kinetics of both activation and desensitization are slower for the insensitive $\alpha 1$ (2$14,60-81) / \alpha 7$ chimera, even though its $\mathrm{EC}_{50}$ is 13 -fold greater than that for $\alpha 7$ and 132-fold greater than that for the sensitive $\alpha 1(1-32,60-81) / \alpha 7$ chimera (Fig. 3A,C). Binding affinity for $\alpha$ Bgt to the $\alpha 1(2-14,60-81) / \alpha 7$ chimera $(2.79 \pm 0.34 \mathrm{~nm})$ was not significantly different from that to wild-type $\alpha 7$ (2.18 \pm 0.52 $\mathrm{nM})$. These observations are consistent with the hypothesis that the low sensitivity of $\alpha 1(2-14,60-81) / \alpha 7$ results from a low probability of opening when bound by agonist at one or two sites, and an increasing probability of opening when liganded at 3 sites. The rapid desensitization kinetics of $\alpha 7$ AChRs results in underestimation of their sensitivity to activation (Papke and Thinschmidt, 1998), and inhibition of their desensitization moves their dose/ response curves to the left (Hurst et al., 2005). However, that is not what is seen here. Activation of $\alpha 7$ AChRs (or others) by binding of one agonist is very inefficient, binding of two agonists is most efficient at activation, and binding at three or more sites enhances the rate of desensitization thereby inhibiting the response (Papke and Thinschmidt, 1998).

mAb 210 did not block muscle AChR responses (data not shown). In contrast, binding of mAb 210 to the $\alpha 1(2-14,60-$ $81) / \alpha 7$ chimera reduced the response to an $\mathrm{EC}_{50}$ concentration of ACh by 70\%. In this experiment, oocytes were incubated with 250 $\mu \mathrm{g} / \mathrm{ml}$ of $\mathrm{mAb}$ for $1 \mathrm{~h}$ before retesting. As a control, similar exposure to $\mathrm{mAb} 306$, which is directed at the cytoplasmic domain of $\alpha 7$, produced no effect. In muscle AChR, the two $\alpha 1$ subunits are not adjacent and the orientation of the MIRs are away from the central axis of the AChR (Beroukhim and Unwin, 1995). This explains why a single $\mathrm{mAb}$ to the MIR cannot crosslink the two MIR epitopes in a single AChR (Conti-Tronconi et al., 1981). However, the presence of five MIR epitopes in the chimera rather than two in a muscle AChR might allow the mAb to the MIR to crosslink adjacent subunits, perhaps thereby impairing function. The higher affinity with which mAbs can bind most chimeras compared to native $\alpha 1$ AChRs (Table 2) might also be explained by the ability of both mAb binding sites to bind to homopentameric chimeras, rather than only 1 site to bind muscle type AChRs (Conti-Tronconi et al., 1981).

\section{Expression and characterization of $\mathrm{AChBP}$ chimeras}

$\alpha 1(1-30,60-81) / A C h B P$, like wild-type AChBP, is water soluble, sediments on sucrose gradients at $4.9 \mathrm{~S}$, and binds $\alpha \mathrm{Bgt}$ (data not shown). ${ }^{125}$ I labeled chimera was used in immune precipitation studies.

As expected, wild-type AChBP was not bound by any rat mAbs to the MIR (data not shown). The $\alpha 1(1-30,60-81) /$ AChBP chimera showed high affinity binding to all rat MIRreactive $m A b s$ tested as well as the human $\mathrm{mAb} 637$, as shown in Table 2 . In contrast to the $\alpha 1(1-32,60-81) / \alpha 7$ chimera, which contained the same $\alpha 1$ sequences, this AChBP chimera was bound by mAb 192 with subnanomolar affinity (Table 2). This suggests that the rest of $\mathrm{AChBP}$ caused conformational changes in the mAb 192 epitope that allowed the binding of mAb 192. This might involve different interactions between the $\alpha 1$ 15-30 sequence and adjacent AChBP sequences. The small differences between affinities of rat mAbs to $\alpha 1 / \alpha 7$ and $\alpha 1 / \mathrm{AChBP}$ chimeras may also result from subtle conformation differences between $\alpha 1$ sequences in $\alpha 1 / \alpha 7$ chimeras and $\alpha 1 / \mathrm{AChBP}$ chimeras.

The only other region which is near the classic MIR loop on the structure of AChBP is the loop between segments $\beta 5$ and $\beta 6$, which corresponds to $\alpha 1107-115$ (Fig. 1A). Including the sequence $\alpha 1$ 107-115 did not improve the binding of mAbs to the MIR (Table 2). Thus the $\beta 5-\beta 6$ loop does not appear to contribute to the antigenic structure of the MIR.

Binding of either nicotine $(1 \mathrm{mM})$ or $\alpha \mathrm{Bgt}(2.5 \mu \mathrm{M})$ to the $\alpha 1 /$ AChBP chimeras decreased binding affinity of $\mathrm{mAb} 637$ slightly $\left(\mathrm{K}_{\mathrm{D}}=0.075 \mathrm{~nm}\right.$ with no cholinergic ligand, $0.18 \mathrm{~nm}$ with nicotine and $0.22 \mathrm{~nm}$ with $\alpha \mathrm{Bgt}$ ) (Fig. 4). Binding affinity of $\mathrm{mAb}$ 192 was also reduced slightly $\left(K_{D}=0.14 \mathrm{~nm}\right.$ with no cholinergic ligand, $0.23 \mathrm{~nm}$ with nicotine and $0.29 \mathrm{nM}$ with $\alpha \mathrm{Bgt})$. Thus, the differences between resting, activated and desensitized conformations do not appear to greatly alter the epitopes within the MIR.

\section{Expression of $\alpha 7(66-76) / \alpha 1$ AChR chimeras}

Since neither $\alpha 1 / \alpha 7$ chimeras nor $\alpha 1 / \mathrm{AChBP}$ chimeras incorporating only the $\mathrm{N}$-terminal sequence $\alpha 12-14$ or $1-32$ were able to form mature pentamers (Fig. $2 A$ ), it was still unknown whether the $\mathrm{N}$-terminal sequence alone contains the epitopes recognized by mAb 637 or 192 or whether parts of $\alpha 160-81$ were also required to form the epitopes. To answer this question, a chimera in which the MIR loop formed by $\alpha 166-76$ was replaced in $\alpha 1$ by corresponding parts of the human neuronal $\alpha 7$ AChR was expressed in oocytes. The $\alpha 7(66-76) / \alpha 1$ chimeric subunit in combination with $\beta 1, \delta$, and $\gamma$ or $\varepsilon$ subunits was able to form functional AChRs on the oocyte surface in either adult $(\varepsilon)$ or fetal $(\gamma)$ forms (Fig. 5A). The $\mathrm{EC}_{50}$ for ACh of the wild type (71 \pm 7 $\mu \mathrm{M})$ and chimeric type $(60 \pm 6 \mu \mathrm{M})$ were similar in the adult form. In the fetal form, wild type $(27 \pm 3 \mu \mathrm{M})$ had somewhat greater sensitivity to ACh than the chimeric type $(65 \pm 8 \mu \mathrm{M})$. The fetal AChR with mutant $\alpha 1$ desensitized more rapidly than 



Figure 2. $\alpha 1 / \alpha 7$ chimeras were more efficiently expressed than wild-type $\alpha 7$ AChRs. $A$, Expression on the oocyte surface detected by binding of ${ }^{125} \mid \alpha B$ gt to intact oocytes is shown. Presence of the $\alpha 1$ MIR increased assembly of mature $\alpha 1 / \alpha 7$ AChRs as measured by the total amount of chimeric $A C h R s$ on the cell surface. Only mature pentameric $A C h R s$ are expected to be transported to the cell surface. All pairs of values shown are significantly different from each other ( $p<0.05)$ except $\alpha 1(66-76) / \alpha 7$ and $\alpha 1(1-32,60-81) / \alpha 7$. B, Similar amounts of wild-type $\alpha 7$ and $\alpha 1 / \alpha 7$ chimera proteins were synthesized as measured by a Western blot (top). However, the $\alpha 1 / \alpha 7$ chimera which forms the most antigenic MIR structure was assembled much more efficiently into mature AChRs expressed on the oocyte surface (bottom, $p<$ 0.05 for all pairs). A chimera containing only $\alpha 11-32$ was not expressed on the cell surface at all. C, The fractions of wild-type $\alpha 7$ and $\alpha 1(2-14,60-81) / \alpha 7$ which could be adsorbed by $\alpha$ Bgt-coupled beads were compared on a Western blot using mAb 319 followed by ${ }^{125}$ I labeled goat anti-rat lgG. Radioactive bands were cut out and quantitated using a $\gamma$ counter. Only mature pentameric AChRs are capable of binding $\alpha$ Bgt. With wild-type $\alpha 7,6.6 \%$ of subunits were assembled into mature AChRs that were adsorbed to $\alpha$ Bgt-coupled beads. Of $\alpha 1(2-14$, $60-81) / \alpha 7$ subunits, $71 \%$ formed mature AChRs. The $\alpha 1(2-14,60-81) / \alpha 7$ chimera always
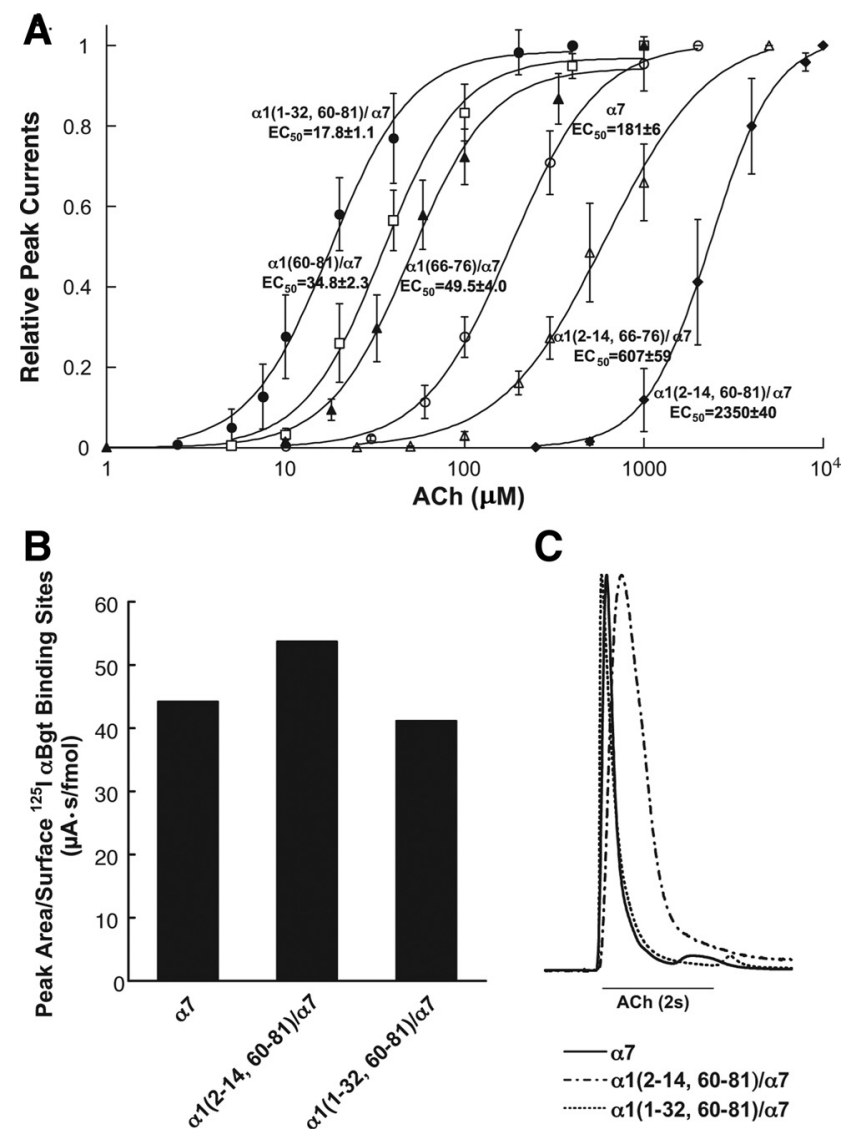

Figure 3. $\alpha 1 / \alpha 7$ chimeras greatly influence the sensitivity to activation of chimeric AChRs by ACh. A, Dose/response curves for ACh show that these chimeras exhibited significantly different sensitivities to activation by ACh. Normalized currents are plotted against agonist concentrations. Averaged data (each point from three to five experiments) were fitted by the Hill equation. The single-fit errors were shown. $\boldsymbol{B}$, The $\alpha 1$ sequences in the chimeras did not change the maximum current per $\mathrm{AChR}$ at saturating $\mathrm{ACh}$ concentrations of chimeric $\mathrm{AChRs}$. Chimeras were expressed in Xenopus oocytes and function was studied with whole-cell recordings using a standard two-microelectrode voltage clamp. The total amount of chimeric AChRs on the cell surface was detected by binding of ${ }^{125} \mid \alpha B g$ t to intact oocytes. C, Time course of currents induced by $\mathrm{EC}_{50}$ concentrations of $\mathrm{ACh}$. Currents were normalized to the peak currents for each chimera.

fetal AChR with native $\alpha 1$, suggesting that $\alpha 1 \mathrm{MIR}$, interacting with $\gamma$, influenced conformation changes associated with desensitization. The $\alpha 7(66-76) / \alpha 1$ AChRs solubilized in Triton X-100 could only be immunoprecipitated by mAb 192, but not by any other classic rat MIR mAbs (like mAbs 198 and 210) or human mAb 637 (Fig. 5B). This confirmed that most mAbs to the MIR absolutely require the $\alpha 166-76$ MIR loop for binding (Saedi et al., 1990), but mAb 192 does not.

The MIR loop was required for binding of 57\% of MG autoantibodies, which corresponds closely to the $55 \%$ of serum antibodies inhibited from binding by mAb 35 in the MG sera tested (Fig. 5C). Replacing the $\alpha 1 \mathrm{MIR}$ did not prevent binding of mAb 192, although it competes with mAb 35 (Tzartos et al., 1998). The sequence 1-32 may contain part or all of the epitope for mAb 192 because this sequence contains rat-specific sequences which may account for the low affinity of mAb 192 for rat $\alpha 1$ AChRs. mAb

$\leftarrow$

had more protein on Western blots than wild-type $\alpha 7$. One possible explanation is that, as with $\alpha 1$ (Merlie and Lindstrom, 1983), unassembled subunits turn over much more rapidly than mature AChRs. Thus the remarkable assembly efficiency of $\alpha 1(2-14,60-81) / \alpha 7$ (Fig. 2A) results in accumulation of much more total $\alpha 7$ subunits. 


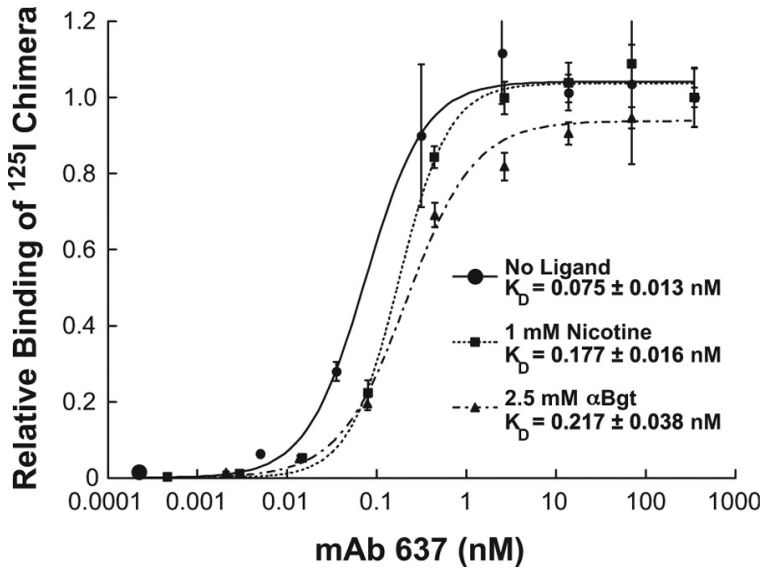

Figure 4. Binding affinity of mAb 637 to the $\alpha 1(1-30,60-81) / A C h B P$ chimera is slightly decreased in the presence of saturating nicotine or $\alpha \mathrm{Bgt}$. The binding of various concentrations of mAbs to ${ }^{125}$ I labeled $\alpha 1 / \mathrm{AChBP}$ chimera was determined by immunoprecipitation in the absence (circles) and presence of either $1 \mathrm{~mm}$ nicotine (square) or $2.5 \mu \mathrm{m} \alpha \mathrm{Bgt}$ (triangle). The averages of duplicate determinations were fitted by the Hill equation to determine $K_{D}$ values (see Table 2).

192 is an absolutely conformation-dependent antibody, which bound $\alpha 1(1-30,60-81) / A C h B P$, but not $\alpha 1(1-32,60-81) / \alpha 7$. Thus, the fact that the $\alpha 7(66-76) / \alpha 1$ AChRs bound mAb 192 suggests that the $\alpha 7$ MIR loop did not significantly alter the conformation of other parts of $\alpha 1$.

Changes in MIR conformation during assembly of $\alpha 1$ AChRs Assembly of human $\alpha 1$ subunits into mature AChRs causes conformation changes which increase the binding affinity of mAbs to the MIR (Merlie and Lindstrom, 1983; Conroy et al., 1990). The human rhabdomyosarcoma cell line TE671 expresses fetal muscle $(\alpha 1 \gamma)(\alpha 1 \delta) \beta 1$ AChRs and a nearly equal amount of monomeric $\alpha 1$ subunits (Conroy et al., 1990). Both mature AChRs and $\alpha 1$ bind ${ }^{125} \mathrm{I} \alpha \mathrm{Bgt}$, although $\alpha 1$ has fivefold lower affinity. Only mature AChRs bind small cholinergic ligands, because the ACh binding sites are formed at the interfaces between $\alpha 1$ and $\delta, \gamma$ or $\varepsilon$ subunits. Thus, in the presence of a high concentration of nicotine, ${ }^{125} \mathrm{I} \alpha$ Bgt is not bound to mature AChRs in TE671 extracts, but only to free $\alpha 1$. Using this approach to distinguish unassembled $\alpha 1$ and mature AChRs, it was shown that the conformationdependent rat $\mathrm{mAb} 35$ reacted 20 -fold better with mature AChRs than with unassembled $\alpha 1$, while the less conformation-dependent mAb 210 bound mature AChRs only fivefold better (Conroy et al., 1990). A selection of $45 \mathrm{MG}$ patient sera reacted on average 14-fold better with mature AChRs than unassembled $\alpha 1$.

Here we took a similar approach to assaying the binding of the highly conformation-dependent human mAb 637, and found that it had $1 \times 10^{4}$ greater reaction with TE671 AChRs than with unassembled $\alpha 1$ (Fig. 6). Thus, the epitope of the MG mAb within the MIR is remarkably more dependent on the native conformation than are either of the rat mAbs 35 or 210 to the MIR. High affinity binding of $\mathrm{mAb} 637$ to $\alpha 1 / \alpha 7$ and $\alpha 1 / \mathrm{AChBP}$ chimeras is strong evidence for how close the conformations of the $\alpha 1$ MIR in these chimeras approaches that of human muscle AChRs.

The $\alpha 1$ MIR expressed in chimeric AChBP was recognized by MG patient sera

Sixty-one randomly chosen MG patient sera archived for several decades were tested for their ability to bind ${ }^{125} \mathrm{I} \alpha 1(1-30,60-81) /$


$\square$ mAb 35 competition
Binding of MG sera to $\alpha 7(66-76) / \alpha 1$ chimeric AChRs

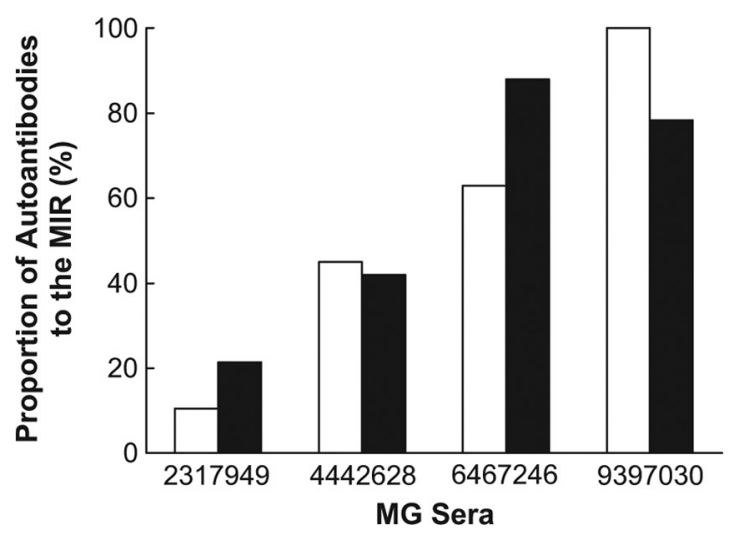

Figure 5. Chimeric $\alpha 7(66-76) / \alpha 1$ AChRs lacking the $\alpha 1$ MIR loop were not immunoprecipitable by most mAbs to the MIR or by many MG patient autoantibodies. $A$, Chimeric $\alpha 7$ (6676)/ $\alpha 1$ subunits formed functional AChRs when coexpressed with $\beta 1, \delta$, and either $\gamma$ or $\varepsilon$ subunits. Chimeric $\alpha 7(66-76) / \alpha 1$ subunits expressed with $\gamma$ subunits to produce a fetal type AChR desensitized more rapidly than wild-type fetal AChR. $B,{ }^{125} \mid \alpha$ Bgt labeled $(\alpha 7(66-76) /$ $\alpha 1)_{2} \beta 1 \delta \gamma$ AChRs were immunoprecipitated from Triton X-100 extracts by mAbs 61 and 192 , but not by mAbs 198, 210 or $637\left({ }^{*} p<0.05\right)$. mAb 61 is directed at the large cytoplasmic domain of the $\alpha 1$ subunit (Ratnam et al., 1986). C, The chimeric $(\alpha 7(66-76) / \alpha 1)_{2} \beta 1 \delta \gamma \mathrm{AChR}$ prevented binding of an average of $57 \%$ of tested MG sera when compared to wild-type $(\alpha 1)_{2} \beta 1 \delta \gamma$. It is consistent with inhibition of binding of these sera to AChR by mAb 35 (average $55 \%)$. The sera are numbered as in Figure $7 B$.

AChBP. Crossreaction with parts of the AChBP sequences other than the MIR was determined using ${ }^{125} \mathrm{I}$ labeled wild-type AChBP and was subtracted from the total binding to the chimeras. Of these, $59 \%$ bound with titers ranging from 0.012 to $19 \mathrm{nM}$, averaging $1.7 \mathrm{~nm}$.

To examine whether the $\beta 5$ - $\beta 6$ loop sequence $\alpha 1$ 107-115 contributes to the epitope recognized by MG autoantibodies, the sixty-one MG patient sera were tested for their ability to bind ${ }^{125} \mathrm{I}$ 


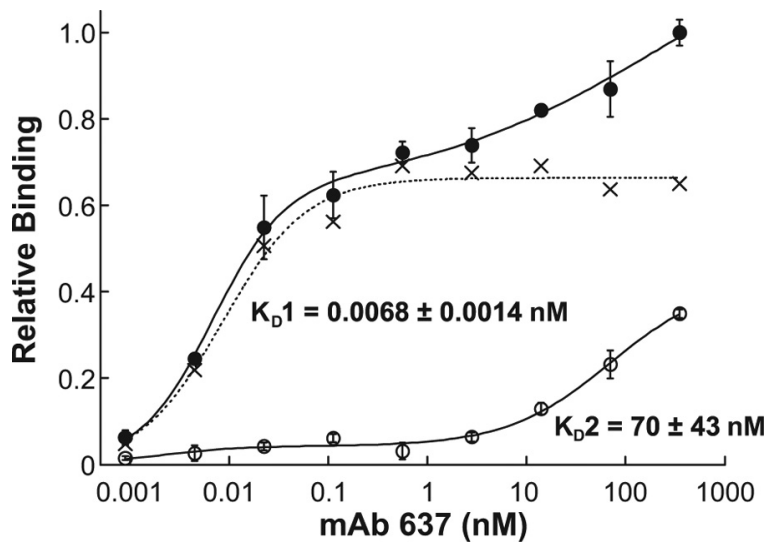

Figure 6. mAb 637 bound to the MIR on unassembled $\alpha 1$ subunits with low affinity compared with native AChR. The binding of different concentrations of mAb to ${ }^{125} \mid \alpha$ Bgt labeled Triton X-100 extracts of TE671 cells was determined by immunoprecipitation assay in the presence (open circles) and absence (closed circles) of $10 \mathrm{~mm}$ nicotine. The binding curve in the absence of nicotine showed a high and a low affinity component. The low affinity phase did not reach a plateau within the tested concentration range. The monotonic calculated high affinity binding curve, determined by subtracting the low affinity binding in the presence of nicotine, is shown as a dotted line ( $X$ symbols). $K_{D} 1$ for high affinity to the MIR in mature AChRs was determined from the monotonic calculated binding curve by fitting with the Hill equation as described in Table $2 . \mathrm{K}_{\mathrm{D}} 2$ for low affinity binding to unassembled $\alpha 1$ was determined from the binding curve in the presence of nicotine by fitting with a two-component Hill equation ( $r=$ $(1-A) /\left(1+10^{(\operatorname{LogKD} 1-\log [A C h]) \times \text { Hill slope } 1}\right)+A /\left(1+10^{(\operatorname{LogKD2}-\log [A C h]) \times \text { Hill slope2 }}\right)$, where $A$ is the proportion of the low affinity component). The binding curve in the absence of nicotine was also fit with this two-component Hill equation. Nicotine inhibits binding of ${ }^{125}$ I $\alpha$ Bgt to mature AChRs but not to the unassembled $\alpha 1$ subunits which are present in these extracts. Thus, in the presence of nicotine binding to only unassembled $\alpha 1$ is assayed.

labeled $\alpha 1 /$ AChBP chimeras. $\alpha 1(1-30,60-81,107-115) / A C h B P$ was not bound by MG sera significantly better than was $\alpha 1$ (1-30, $60-81$ )/AChBP (Fig. $7 A$ ). Thus, the $\beta 5-\beta 6$ loop does not contribute to MIR epitopes recognized by MG patient sera, just as it dose not contribute to binding of mAbs to the MIR (Table 2).

A collection of recently collected MG sera was used to assay both the fraction of autoantibodies which could bind to the MIR in the $\alpha 1(1-30,60-81) / A C h B P$ chimera and the fraction which could be inhibited from binding to human $\alpha 1$ AChR by mAb 35 to the MIR (Fig. $7 B$ ). The chimera adsorbed an average of $15 \%$ of the autoantibodies. $\mathrm{mAb} 35$ inhibited the binding of an average of $63 \%$ of the autoantibodies. These results are consistent with the concepts that: (1) the chimera contains several closely spaced conformation-dependent epitopes which are bound with high affinity by some mAbs and autoantibodies to the MIR, (2) muscle AChR contains more MIR epitopes, the majority of which include the MIR loop, and (3) binding of a mAb to the MIR can inhibit the binding of autoantibodies to many overlapping and closely spaced epitopes within the MIR.

\section{The MIR in the $\alpha 1 / \mathrm{AChBP}$ chimera was recognized by a} majority of feline and canine MG sera

Canine and feline MG are good naturally occurring models for human MG (Shelton and Lindstrom, 2001). mAb 35 inhibited binding of an average of $68 \%$ of the autoantibodies in canine MG (Shelton et al., 1988). Since mAbs to the MIR from rats with EAMG recognize overlapping but distinct epitopes other than those that are recognized by MG patients, we asked whether canine or feline MG autoantibodies bind to the same epitopes recognized by MG patients. To answer this question, 21 canine and 39 feline MG sera were randomly chosen and tested for crossre-


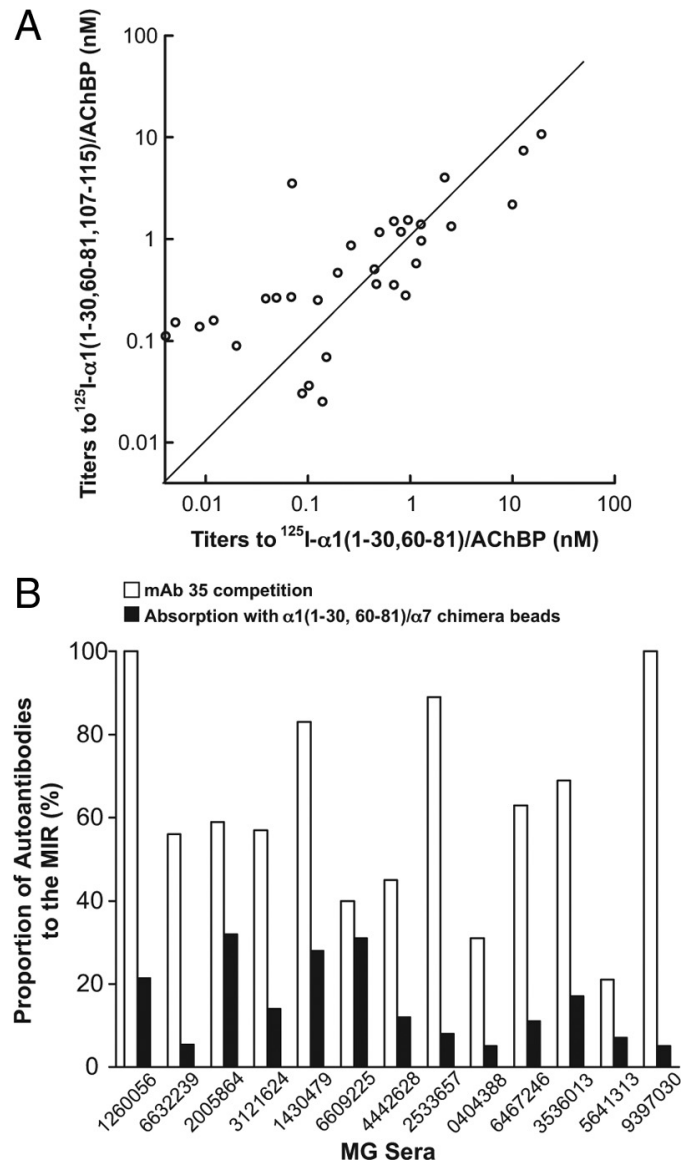

Figure 7. The MIR expressed in chimeric AChBP was recognized by MG patient sera. $A$, Titers of sera from $61 \mathrm{MG}$ patients were determined by immunoprecipitation of ${ }^{125} \mid \alpha 1(1-30,60$ $81) / A C h B P$ or ${ }^{125} \mid \alpha 1(1-30,60-81,107-115) / A C h B P$. A linear regression line was fitted to the data with the following parameters: $r=0.90$ and slope $=0.49$. These results indicated that both chimeras were recognized equally, implying that the sequence $\alpha 1107-115$ did not contribute significantly to autoantibody binding. $\boldsymbol{B}$, The proportion of $M G$ autoantibodies specific to the MIR was determined in sera from 13 other MG patients by two methods. The fraction of autoantibodies to human muscle AChR that could bind to the $\alpha 1(1-30,60-81) / \mathrm{AChBP}$ chimera was determined by pre-adsorbing the antisera with the $\alpha 1(1-30,60-81) / A C h B P$ chimera coupled to agarose. The fraction of autoantibodies that could be inhibited from binding to AChR by the presence of excess mAb 35 was also determined. Binding of mAb 35 inhibited the binding of a greater proportion of the autoantibodies than could bind the $\alpha 1$ epitopes expressed in the chimera.

are shown in Figure 8 . The chimera was recognized by $28 \%$ of canine MG autoantibodies and $24 \%$ of feline MG autoantibodies. Fifty-seven percent of canine MG sera, and $80 \%$ of feline MG sera, had $>5 \%$ crossreactivity with $\alpha 1(1-30,60-81) / \mathrm{AChBP}$ chimera.

\section{Discussion}

The MIR is important because half or more of the autoantibodies to AChRs in EAMG and MG in several species are directed at this region, and because these antibodies contribute to pathology at the neuromuscular junction (Tzartos et al., 1982, 1983; Shelton et al., 1988). Our data showed that two $\alpha 1$ sequences (the MIR loop and the N-terminal $\alpha$ helical region) must interact to form MIR epitopes recognized by mAbs from rats with EAMG or humans with MG. These results are consistent with studies of the crystal structure of mouse AChR $\alpha 1$ subunit extracellular domain, in which the MIR loop is in close association with the N-terminal $\alpha$ helix and the $\beta 5$ - $\beta 6$ turn loop (Dellisanti et al., 2007). Our results 
showed that the $\mathrm{N}$-terminal $\alpha$ helix was critical to the antigenic structure of the MIR, but the $\beta 5-\beta 6$ turn loop (i.e., $\alpha 1$ $107-115)$ was not. Our results further showed that the conformation of the MIR changes when $\alpha 1$ subunits are assembled into mature human AChRs, conferring higher affinities for binding of mAbs to the MIR. Thus, the conformation of crystallized unassembled $\alpha 1$ subunits (Dellisanti et al., 2007) must differ somewhat from $\alpha 1$ in mature AChR pentamers. However, details of the structure of unassembled $\alpha 1$ subunits are consistent with our observations. Amino acids 68 and 71, which are critical to the antigenic structure of the MIR loop (Saedi et al., 1990), pack closely with amino acids of the N-terminal $\alpha$ helix (Dellisanti et al., 2007).

The $\alpha 1$ MIR loop is critical for binding of most antibodies to the MIR. Replacing the MIR loop in $\alpha 1$ subunits assembled in $\alpha 1$ AChRs with the corresponding $\alpha 7$ loop inhibited the binding of MG autoantibodies in the same large proportion that they are inhibited by binding of $\mathrm{mAb} 35$. Although the $\alpha 1(1-30,60-81) / \mathrm{AChBP}$ chimera was recognized by $59 \%$ of human MG sera, the proportion of autoantibodies bound to this chimera was still lower than the proportion of autoantibody binding blocked by mAb 35 .

MG sera and mAbs bind to unassembled $\alpha 1$ with much lower affinity than to $\alpha 1$ assembled in mature AChRs (Conroy et al., 1990). This could reflect a conformation change in $\alpha 1$ on assembly. Alternatively, or in addition, contributions to the MIR from adjacent subunits could explain why $\alpha 1$ /ACHBP MIR chimeras adsorb a much smaller fraction of MG patient autoantibodies than are prevented from binding by $\mathrm{mAb} 35$. Construction of more complex AChBP chimeras containing both $\alpha 1$ and other subunit sequences will be required to test this possibility.

$\alpha 1$ subunits differ greatly from both $\alpha 7$ and AChBP in the sequences $1-14$ and 15-32 (Fig. 1C). Thus, it is not surprising that a1 1-14 or 15-32 are incompatible with forming mature AChRs alone in $\alpha 7$ chimeras. The interactions between $\alpha 11-14$ and 66-76 permit assembly of this domain with $\alpha 7$, while conferring a particular conformation on the domain with high affinity for conformation-dependent mAbs. The stability of this domain helps to overcome the incompatibility of 15-32 with $\alpha 7$, but leaves the $\mathrm{AChR}$ in a conformation which is more easily activated by agonists. The 15-32 sequence apparently assumes a different conformation in association with adjacent AChBP sequences than $\alpha 7$ sequences, because the $\alpha 1(1-30,60-81) / \mathrm{AChBP}$ permits binding of $\mathrm{mAb} 192$ and $\alpha 1(1-32,60-81) / \alpha 7$ does not.

MG in dogs and cats provide a good animal models for human MG (Shelton et al., 1988, 2000). A majority of feline (80\%), and canine (57\%) MG sera bind to a chimera forming MIR epitopes recognized by human MG patients.

The rat mAb 210 to the MIR recognizes human $\alpha 3$ (Wang et al., 1998) but not rodent $\alpha 3$ (Feng et al., 1998) because of two amino acid differences (amino acids 68 and 73) in the MIR loop order of titers against muscle AChR.
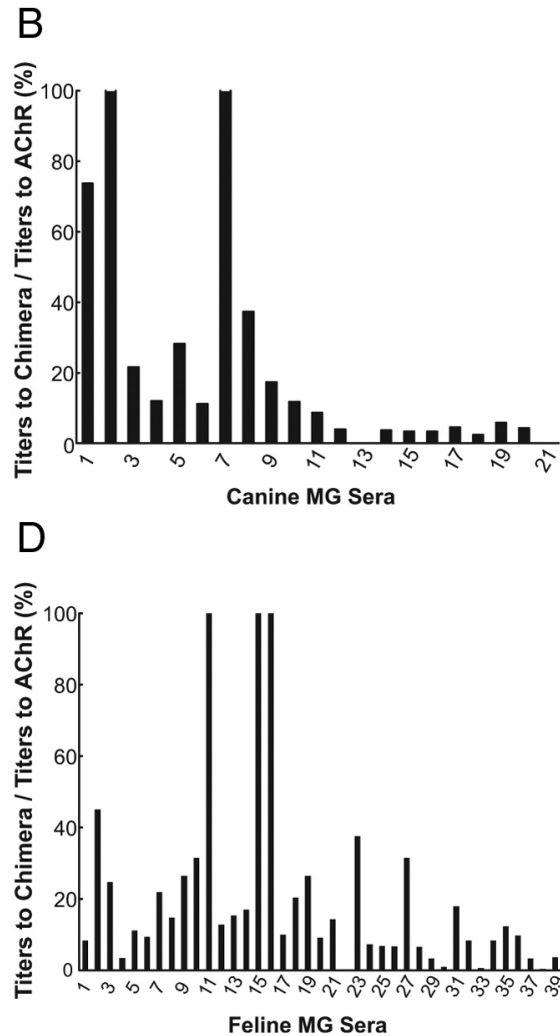

eline MG Sera

Figure 8. The MIR in the $\alpha 11-30,60-81 / \mathrm{AChBP}$ chimera was recognized by a majority of feline and canine MG sera. $A, C$, The apparent titers of 21 canine and 39 feline MG sera against ${ }^{125}$ I labeled $\alpha 1(1-30,60-81) / A C h B P$ were determined by immuno-

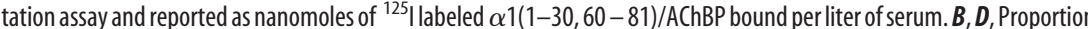
(n) relative to the corresponding titer against muscle $A C h R$ from that species. From left to right, $M G$ sera were arranged in ascending

between human $\alpha 3$ and rodent $\alpha 3$. Changing these two amino acids in rat $\alpha 3$ to their human $\alpha 3$ equivalents allows mAb 210 to bind rodent $\alpha 3$ (Ficklin et al., 2005). Rat mAbs to the MIR may recognize epitopes slightly different from those recognized by humans, because $\mathrm{B}$ cells which produce antibodies that can react with the human $\alpha 3$ epitope are not eliminated.

mAb 192 competes for binding with mAb 35 (Tzartos et al., 1998). Its binding did not require the MIR loop upon which binding of $\mathrm{mAb} 35$ is dependent. This can be explained by the observation that mAbs can obscure large areas $(750 \AA)$ on proteins to which they are bound (Mariuzza et al., 1987; Konstantakaki et al., 2007).

Fostieri et al. (2005) reported another example of the heterogeneity of MIR epitopes. A Fab derived from a MG patient, sh6.4, inhibited binding of $\mathrm{mAb} 35$ and 198, but not $\mathrm{mAb}$ 192. sh6.4 did not bind free $\alpha 1$ subunits, but it did bind human $\alpha \gamma$ and $\alpha \varepsilon$ dimers. Some MIR epitopes may contain some parts of neighboring subunits. This could explain the higher affinities of mAbs for AChRs as compared to monomeric $\alpha 1$ subunits (Conroy et al., 1990). The absence of parts of the MIR formed by adjacent subunits in the $\alpha 1 / \mathrm{AChBP}$ chimeras may reduce binding of some MG autoantibodies.

$\alpha 1 / \alpha 7$ chimeras expressed on the oocyte surface retain their ability to function as AChRs. The sensitivity to activation of chimeric AChRs by ACh was greatly influenced by the $\alpha 1$ sequences within them. Addition of the sequence 15-32 to the $\alpha 1(2-14$, $60-81) / \alpha 7$ chimera resulted not only in binding of the MG patient $\mathrm{mAb} 637$ but also in a 130 -fold increase in sensitivity to 
activation by ACh. This suggests that interaction between parts of the sequences that form the conformation-dependent MIR may influence conformational changes in this region associated with activation (Henchman et al., 2003). Although closing of the C loop over the ACh binding site when agonists bind is a critical conformational change associated with activation (Hansen et al., 2005), and is propagated through the AChR to regulate opening of the distant channel gate, AChR activation must be associated with conformation changes throughout the AChR. For example, changing accessory subunits (like $\beta 1$ of muscle AChRs), which do not participate in forming ACh binding sites, has large effects on the sensitivity of AChRs to activation (Kuryatov et al., 2008). The decreased binding affinity of mAbs in the presence of either nicotine or $\alpha$ Bgt suggests small effects on the conformation of the MIR with a desensitized state or a resting state. Also, the $\alpha 7(66-$ 76) $/ \alpha 1$ chimera desensitized more rapidly than wild-type $\alpha 1$. Binding of mAb 210 to the $\alpha 1(2-14,60-81) / \alpha 7$ chimera reduced activation by $70 \%$, consistent with there being some conformational feedback between the MIR and the ACh binding sites.

Disrupting the structure of the MIR can disrupt conformational maturation of $\alpha 1$ subunits and expression of $\alpha 1$ AChRs. Incorporation of the 25 amino acid P3A exon between amino acids 58 and 59 of human AChR $\alpha 1$ subunits prevents expression of the AChR (Newland et al., 1995).

The importance of the MIR in conformational maturation is demonstrated by our observation that incorporation of the $\alpha 1$ MIR into $\alpha 7$ greatly increases chimeric subunit conformational maturation and expression of mature AChRs. Efficient assembly of $\alpha 7$ AChRs has been difficult to achieve from cloned subunits (Gee et al., 2007). In $\alpha 1$ subunits, conformational maturation of the MIR and the ACh binding site (detected by the ability to bind $\mathrm{mAb} 35$ and $\alpha$ Bgt, respectively) occur simultaneously before assembly (Merlie and Lindstrom, 1983). Conformational maturation of chimeric $\alpha 1 / \alpha 7$ subunits may be nucleated by the association of the N-terminal and MIR loop components of the MIR, and this may drive efficient assembly of these chimeric AChRs. One of the normal functional roles of the MIR may be to nucleate conformational maturation of unassembled subunits.

Recently Castillo et al. (2009) found that deleting or disrupting the N-terminal $\alpha$ helix of $\alpha 7, \alpha 3, \alpha 4, \beta 2$ or $\beta 4$ AChR subunits or $5-\mathrm{HT}_{3 \mathrm{~A}}$ subunits prevented assembly of mature AChRs. In congenital myasthenic syndromes, some mutations in or near the $\mathrm{N}$-terminal $\alpha$ helix and MIR loop in the AChR subunits reduce expression of AChR (Engel and Sine, 2005). These observations suggest that the importance of the interaction between the N-terminal $\alpha$ helix and MIR loop, for conformational maturation and assembly, which we have demonstrated in $\alpha 1 / \alpha 7$ chimeras, is a general phenomenon applying to all subunits of AChRs and related receptors. Transmembrane domain interactions and cytoplasmic regions adjacent to the transmembrane domains can also influence expression and function of $\alpha 7$ AChRs (Castelán et al., 2007; Gee et al., 2007).

In the future, $\mathrm{x}$-ray crystallography of mAbs bound to the MIR in $\alpha 1$ /AChBP chimeras should allow us to determine the structure of the MIR at atomic resolution. These chimeras should also permit testing the ability of a native $\alpha 1$ MIR structure to induce or inhibit EAMG.

\section{References}

Beroukhim R, Unwin N (1995) Three-dimensional location of the main immunogenic region of the acetylcholine receptor. Neuron 15:323-331.

Castelán F, Mulet J, Aldea M, Sala S, Sala F, Criado M (2007) Cytoplasmic regions adjacent to the M3 and M4 transmembrane segments influence expression and function of $\alpha 7$ nicotinic acetylcholine receptors. A study with single amino acid mutants. J Neurochem 100:406-415.

Castillo M, Mulet J, Aldea M, Gerber S, Sala S, Sala F, Criado M (2009) Role of the $\mathrm{N}$-terminal $\alpha$-helix in biogenesis of $\alpha 7$ nicotinic receptors. J Neurochem 108:1399-1409.

Colman A (1984) Transcription and translation: a practical approach. In: Practical approach series. (Hames BD, Higgins SJ, eds), pp 271-302. Oxford, UK: IRL Press.

Conroy WG, Saedi MS, Lindstrom J (1990) TE671 cells express an abundance of a partially mature acetylcholine receptor $\alpha$ subunit which has characteristics of an assembly intermediate. J Biol Chem 265:2164221651.

Conti-Tronconi B, Tzartos S, Lindstrom J (1981) Monoclonal antibodies as probes of acetylcholine receptor structure. 2. Binding to native receptor. Biochemistry 20:2181-2191.

Das MK, Lindstrom J (1989) The main immunogenic region of the nicotinic acetylcholine receptor: interaction of monoclonal antibodies with synthetic peptides. Biochem Biophys Res Commun 165:865-871.

Dellisanti CD, Yao Y, Stroud JC, Wang ZZ, Chen L (2007) Crystal structure of the extracellular domain of nAChR $\alpha 1$ bound to $\alpha$-bungarotoxin at $1.94 \AA$ resolution. Nat Neurosci 10:953-962.

Engel AG, Sine SM (2005) Current understanding of congenital myasthenic syndromes. Curr Opin Pharmacol 5:308-321.

Feng G, Steinbach JH, Sanes JR (1998) Rapsyn clusters neuronal acetylcholine receptors but is inessential for formation of an interneuronal cholinergic synapse. J Neurosci 18:4166-4176.

Ficklin MB, Zhao S, Feng G (2005) Ubiquilin-1 regulates nicotine-induced up-regulation of neuronal nicotinic acetylcholine receptors. J Biol Chem 280:34088-34095.

Fostieri E, Tzartos SJ, Berrih-Aknin S, Beeson D, Mamalaki A (2005) Isolation of potent human Fab fragments against a novel highly immunogenic region on human muscle acetylcholine receptor which protect the receptor from myasthenic autoantibodies. Eur J Immunol 35:632-643.

Gee VJ, Kracun S, Cooper ST, Gibb AJ, Millar NS (2007) Identification of domains influencing assembly and ion channel properties in $\alpha 7$ nicotinic receptor and $5-\mathrm{HT}_{3}$ receptor subunit chimaeras. $\mathrm{Br} \mathrm{J}$ Pharmacol 152:501-512.

Gerzanich V, Wang F, Kuryatov A, Lindstrom J (1998) $\alpha 5$ subunit alters desensitization, pharmacology, $\mathrm{Ca}^{++}$permeability and $\mathrm{Ca}^{++}$modulation of human neuronal $\alpha 3$ nicotinic receptors. J Pharmacol Exp Ther 286:311-320.

Graus YF, de Baets MH, van Breda Vriesman PJ, Burton DR (1997) Antiacetylcholine receptor Fab fragments isolated from thymus-derived phage display libraries from myasthenia gravis patients reflect predominant specificities in serum and block the action of pathogenic serum antibodies. Immunol Lett 57:59-62.

Gullick WJ, Lindstrom JM (1983) Mapping the binding of monoclonal antibodies to the acetylcholine receptor from Torpedo californica. Biochemistry 22:3312-3320.

Hansen SB, Radic' Z, Talley TT, Molles BE, Deerinck T, Tsigelny I, Taylor P (2002) Tryptophan fluorescence reveals conformational changes in the acetylcholine binding protein. J Biol Chem 277:41299-41302.

Hansen SB, Talley TT, Radic Z, Taylor P (2004) Structural and ligand recognition characteristics of an acetylcholine-binding protein from Aplysia californica. J Biol Chem 279:24197-24202.

Hansen SB, Sulzenbacher G, Huxford T, Marchot P, Taylor P, Bourne Y (2005) Structures of Aplysia AChBP complexes with nicotinic agonists and antagonists reveal distinctive binding interfaces and conformations. EMBO J 24:3635-3646.

Henchman RH, Wang HL, Sine SM, Taylor P, McCammon JA (2003) Asymmetric structural motions of the homomeric $\alpha 7$ nicotinic receptor ligand binding domain revealed by molecular dynamics simulation. Biophys J 85:3007-3018.

Hurst RS, Hajós M, Raggenbass M, Wall TM, Higdon NR, Lawson JA, Rutherford-Root KL, Berkenpas MB, Hoffmann WE, Piotrowski DW, Groppi VE, Allaman G, Ogier R, Bertrand S, Bertrand D, Arneric SP (2005) A novel positive allosteric modulator of the $\alpha 7$ neuronal nicotinic acetylcholine receptor: in vitro and in vivo characterization. J Neurosci 25:4396-4405.

Im SH, Barchan D, Souroujon MC, Fuchs S (2000) Role of tolerogen conformation in induction of oral tolerance in experimental autoimmune myasthenia gravis. J Immunol 165:3599-3605. 
Konstantakaki M, Tzartos SJ, Poulas K, Eliopoulos E (2007) Molecular modeling of the complex between Torpedo acetylcholine receptor and anti-MIR Fab198. Biochem Biophys Res Commun 356:569-575.

Kontou M, Leonidas DD, Vatzaki EH, Tsantili P, Mamalaki A, Oikonomakos NG, Acharya KR, Tzartos SJ (2000) The crystal structure of the Fab fragment of a rat monoclonal antibody against the main immunogenic region of the human muscle acetylcholine receptor. Eur J Biochem 267: 2389-2397.

Kuryatov A, Gerzanich V, Nelson M, Olale F, Lindstrom J (1997) Mutation causing autosomal dominant nocturnal frontal lobe epilepsy alters $\mathrm{Ca}^{++}$ permeability, conductance, and gating of human $\alpha 4 \beta 2$ nicotinic acetylcholine receptors. J Neurosci 17:9035-9047.

Kuryatov A, Onksen J, Lindstrom J (2008) Roles of accessory subunits in $\alpha 4 \beta 2^{\star}$ nicotinic receptors. Mol Pharmacol 74:132-143.

Lennon VA, Lambert EH, Leiby KR, Okarma TB, Talib S (1991) Recombinant human acetylcholine receptor $\alpha$-subunit induces chronic experimental autoimmune myasthenia gravis. J Immunol 146:2245-2248.

Lindstrom J (1996) Neuronal nicotinic acetylcholine receptors. Ion Channels 4:377-450.

Lindstrom JM (2000) Acetylcholine receptors and myasthenia. Muscle Nerve 23:453-477.

Lindstrom J, Einarson B (1979) Antigenic modulation and receptor loss in experimental autoimmune myasthenia gravis. Muscle Nerve 2:173-179.

Lindstrom J, Einarson B, Merlie J (1978) Immunization of rats with polypeptide chains from Torpedo acetylcholine receptor causes an autoimmune response to receptors in rat muscle. Proc Natl Acad Sci U S A 75:769-773.

Mariuzza RA, Phillips SE, Poljak RJ (1987) The structural basis of antigenantibody recognition. Annu Rev Biophys Biophys Chem 16:139-159.

McLane KE, Wu X, Lindstrom JM, Conti-Tronconi BM (1992) Epitope mapping of polyclonal and monoclonal antibodies against two $\alpha$-bungarotoxin-binding $\alpha$ subunits from neuronal nicotinic receptors. J Neuroimmunol 38:115-128.

Merlie JP, Lindstrom J (1983) Assembly in vivo of mouse muscle acetylcholine receptor: identification of an $\alpha$ subunit species that may be an assembly intermediate. Cell 34:747-757.

Newland CF, Beeson D, Vincent A, Newsom-Davis J (1995) Functional and non-functional isoforms of the human muscle acetylcholine receptor. J Physiol 489:767-778.

Papke RL, Thinschmidt JS (1998) The correction of $\alpha 7$ nicotinic acetylcholine receptor concentration-response relationships in Xenopus oocytes. Neurosci Lett 256:163-166.

Peng X, Gerzanich V, Anand R, Whiting PJ, Lindstrom J (1994) Nicotineinduced increase in neuronal nicotinic receptors results from a decrease in the rate of receptor turnover. Mol Pharmacol 46:523-530.

Ratnam M, Nguyen DL, Rivier J, Sargent PB, Lindstrom J (1986) Transmembrane topography of nicotinic acetylcholine receptor: immunochemical tests contradict theoretical predictions based on hydrophobicity profiles. Biochemistry 25:2633-2643.

Reeves PJ, Callewaert N, Contreras R, Khorana HG (2002) Structure and function in rhodopsin: high-level expression of rhodopsin with restricted and homogeneous $\mathrm{N}$-glycosylation by a tetracycline-inducible
$\mathrm{N}$-acetylglucosaminyltransferase I-negative HEK293S stable mammalian cell line. Proc Natl Acad Sci U S A 99:13419-13424.

Saedi MS, Anand R, Conroy WG, Lindstrom J (1990) Determination of amino acids critical to the main immunogenic region of intact acetylcholine receptors by in vitro mutagenesis. FEBS Lett 267:55-59.

Shelton GD, Lindstrom JM (2001) Spontaneous remission in canine myasthenia gravis: implications for assessing human MG therapies. Neurology 57:2139-2141.

Shelton GD, Cardinet GH, Lindstrom JM (1988) Canine and human myasthenia gravis autoantibodies recognize similar regions on the acetylcholine receptor. Neurology 38:1417-1423.

Shelton GD, Ho M, Kass PH (2000) Risk factors for acquired myasthenia gravis in cats: 105 cases (1986-1998). J Am Vet Med Assoc 216:55-57.

Tzartos S, Langeberg L, Hochschwender S, Lindstrom J (1983) Demonstration of a main immunogenic region on acetylcholine receptors from human muscle using monoclonal antibodies to human receptor. FEBS Lett 158:116-118.

Tzartos S, Hochschwender S, Vasquez P, Lindstrom J (1987) Passive transfer of experimental autoimmune myasthenia gravis by monoclonal antibodies to the main immunogenic region of the acetylcholine receptor. J Neuroimmunol 15:185-194.

Tzartos SJ, Lindstrom JM (1980) Monoclonal antibodies used to probe acetylcholine receptor structure: localization of the main immunogenic region and detection of similarities between subunits. Proc Natl Acad Sci U S A 77:755-759.

Tzartos SJ, Rand DE, Einarson BL, Lindstrom JM (1981) Mapping of surface structures of electrophorus acetylcholine receptor using monoclonal antibodies. J Biol Chem 256:8635-8645.

Tzartos SJ, Seybold ME, Lindstrom JM (1982) Specificities of antibodies to acetylcholine receptors in sera from myasthenia gravis patients measured by monoclonal antibodies. Proc Natl Acad Sci U S A 79:188-192.

Tzartos SJ, Loutrari HV, Tang F, Kokla A, Walgrave SL, Milius RP, ContiTronconi BM (1990) Main immunogenic region of Torpedo electroplax and human muscle acetylcholine receptor: localization and microheterogeneity revealed by the use of synthetic peptides. J Neurochem 54:51-61.

Tzartos SJ, Barkas T, Cung MT, Mamalaki A, Marraud M, Orlewski P, Papanastasiou D, Sakarellos C, Sakarellos-Daitsiotis M, Tsantili P, Tsikaris V (1998) Anatomy of the antigenic structure of a large membrane autoantigen, the muscle-type nicotinic acetylcholine receptor. Immunol Rev 163:89-120.

van der Neut Kolfschoten M, Schuurman J, Losen M, Bleeker WK, MartínezMartínez P, Vermeulen E, den Bleker TH, Wiegman L, Vink T, Aarden LA, De Baets MH, van de Winkel JG, Aalberse RC, Parren PW (2007) Anti-inflammatory activity of human IgG4 antibodies by dynamic Fab arm exchange. Science 317:1554-1557.

Vernino S, Lennon VA (2004) Autoantibody profiles and neurological correlations of thymoma. Clin Cancer Res 10:7270-7275.

Wang F, Nelson ME, Kuryatov A, Olale F, Cooper J, Keyser K, Lindstrom J (1998) Chronic nicotine treatment up-regulates human $\alpha 3 \beta 2$ but not $\alpha 3 \beta 4$ acetylcholine receptors stably transfected in human embryonic kidney cells. J Biol Chem 273:28721-28732. 